\title{
The diffuse-type gastric cancer epidemiology enigma
}

\author{
Paulo Pimentel Assumpção', Williams Fernandes Barra ${ }^{1 *} \mathbb{B}$, Geraldo Ishak², Luiz Gonzaga Vaz Coelho ${ }^{3}$, \\ Felipe José Fernandez Coimbra ${ }^{4}$, Helano Carioca Freitas ${ }^{5}$, Emmanuel Dias-Neto ${ }^{6}$, M. Constanza Camargo ${ }^{7}$ and \\ Moyses Szklo 8
}

\begin{abstract}
Background: Intestinal and diffuse gastric adenocarcinomas differ in clinical, epidemiological and molecular features. However, most of the concepts related to the intestinal-type are translated to gastric adenocarcinoma in general; thus, the peculiarities of the diffuse-type are underappreciated.

Results: Besides its growing importance, there are many gaps about the diffuse-type carcinogenesis and, as a result, its epidemiologic and pathogenetic features remain poorly understood.

Conclusions: Alternative hypotheses to explain these features are discussed, including the role of the gastric microbiota, medical therapies, and modifications in the stomach's microenvironment.
\end{abstract}

\section{Background}

Gastric adenocarcinoma (GAC) is a leading cause of cancer-related deaths, although a reduction in incidence has been observed $[1,2]$. Whereas these are different histology entities [3], intestinal and diffuse Lauren's GAC types are jointly considered when interpreting epidemiological data, and when contemplating interventional approaches aiming to reduce gastric cancer (GC) burden $[1,2]$. Since the intestinal-GAC has been historically the most often studied type, data from this tumor are usually extrapolated to the diffuse-GAC. However, when examining and interpreting carcinogens and the epidemiology of diffuse-type carcinogenesis, inconsistences with regard to intestinal-type are evident [3]. These differences should be considered when trying to understand its specific pathogenesis and to apply this knowledge to develop interventional approaches. Our purpose in this commentary is to discuss some of the

\footnotetext{
* Correspondence: ajuru2@gmail.com

${ }^{1}$ Núcleo de Pesquisas em Oncologia, Universidade Federal do Pará, Belém, Pará, Brazil

Full list of author information is available at the end of the article
}

issues surrounding the diffuse-type GAC and to offer hypothesis to shed light on these features.

\section{Epidemiology}

GC was responsible for more than $1,000,000$ new cases in 2018 with an estimated 783,000 deaths, being the fifth most frequent and the third leading cause of cancer death. The global incidence of GC varies markedly, with the highest burden observed in some of the less developed areas of the world. Asia, Eastern Europe and South America account for most GC cases and, thus, for their high mortality rates [1]. GAC is the most common type of GC, being classically classified, according to Lauren, as intestinal- or diffuse-types [2].

GAC incidence has been declining since 1930's [1], mainly due to the marked decrease of the intestinal-type. A decreasing trend of the intestinal- and a stable or increasing trend of the diffuse-type have been found in both high and low GC risk areas $[4,5]$. However, the incidence in Korean Americans has declined during recent years, for both cardia and non-cardia sites and for both intestinal- and diffuse-type histology [6]. Several factors can explain the decreased incidence of GAC, including 
improvement of food preservation following the widespread use electricity and refrigeration replacing the consumption of salt-preserved foods $[1,4,5]$ and, most importantly, the decrease in $H$. pylori infection rates, possibly resulting from better hygiene habits and the broad use of antibiotics [7]. However, the effects of these factors over the incidence of the diffuse-type appear to be flimsy. Indeed, risk of diffuse GAC has been generally attributed to a weaker influence of environmental factors and a stronger relevance of genetic factors [3]. Lauren's intestinal and diffuse histological types have different prognoses. The diffuse-type generally presents reduced survival when compared to the intestinal-type [8].

\section{Etiology}

The knowledge of environmental factors behind GAC has fundamental implications for primary prevention. Whereas case-control studies suggest an inverse association of fruit and vegetables consumption with the risk of GAC in different populations [9], data from prospective studies are less consistent with regard to the protective role of diet [10]. Additionally, the genetic factors attributed to the diffuse-type non-familial GAC remain elusive.

H. pylori infection is recognized by World Health Organization as a necessary cause of non-cardia gastric cancer, irrespective of Lauren's subtype classification [7]. Nevertheless eradication of $H$. pylori seems to reduce the risk of gastric cancer [11], including the decrease in incidence of metachronous tumors among patients that were effectively treated after endoscopic resection of early gastric cancer [12] and also among patients with a family history of gastric cancer [13], there are controversial reports about this theme [14], in special, regarding the risk for diffuse type GC. Take et al (2020) demonstrated an increased risk for diffuse type gastric cancer 10 years after $H$. pylori eradication [15], showing the necessity of maintaining endoscopic follow-up for many years after effective $H$. pylori eradication.

Considering that $H$. pylori prevalence has been reduced both by lower exposure to, and higher level of bacteria eradication, a reduction of both intestinaland diffuse-types would have been expected. However, the reduction of intestinal-type GAC incidence has not been followed by a parallel decrease of the diffuse-type $[4,5]$, which suggests that either $H$. pylori or other risk factors for the intestinal-type are not as strongly related to the diffuse-type [3-5], or that other risk factors to which the latter type is attributed has increased over time, thus, offsetting the decrease of $H$. pylori prevalence. These data strengths the hypothesis about the considered unexplained peculiarities involving diffuse type GC, including the etiological role of $H$. pylori, in diffuse tumors.

\section{Age of occurrence and gender differences}

The diffuse GAC seems to occur at an earlier age than the intestinal GAC $[3,4]$. Since it takes many years for a chronic $H$. pylori infection to cause gastric cancer, the required time for an $H$. pylori-dependent diffuse-type carcinogenesis should be shorter than that for the intestinal-type. A shorter latency for the diffuse-type may suggest the existence of combination of genomic alterations in the host favoring carcinogenesis, and/or distinct virulence of $H$. pylori strains (including eradication-resistant strains) behind the diffuse GAC. Therefore, the H. pylori treatment usually prescribed to adult patients may not be fully beneficial to prevent diffuse-type tumors, since the required molecular steps involved in this type of cancer may have already occurred before bacteria eradication. Consequently, as the prevalence of $H$. pylori infection among children has diminished significantly [16], an accompanying decline in the burden of the diffuse-type GAC would be expected; however, this has not happened so far.

Although the intestinal-type is more frequent in men than in women (2:1), the proportion of the diffuse-type is similar between genders [3, 4]. Environmental risk factors may explain the higher incidence of the intestinaltype among men, since exposure to risk factors, including tobacco, alcohol, and dietary carcinogens, is more frequent in men. On the other hand, genetic factors are thought to be more significant in women. However, none of these hypotheses explaining gender-differences have been confirmed [3].

\section{Location of tumors}

Intestinal-type GAC has a higher incidence in the distal stomach while diffuse-type tumors are more frequently found in proximal regions $[3,4]$. The incidence of distal tumors has diminished, and that of corpus and proximal non-cardia tumors has remained stable [3-5]. The incidence of distal tumors decreased in parallel with the reduction of the intestinal-type GAC. However, as distal tumors are not exclusively from the intestinal-type, at least a modest decrease in diffuse tumors would be expected if both intestinal and diffuse-types had a similar risk factor profile.

\section{Molecular classification}

In addition to presenting phenotypic differences, diffuse GC also have specific molecular profiles, and carcinogenesis features, that differentiate it from the intestinal type. Looking at the molecular profiles of diffuse and intestinal types, there are important differences that strengthen the hypothesis that there are two diverse tumors [17-21].

The molecular characterization performed by "The Cancer Genome Atlas" Research Network (TCGA) divided 
the GC into four subtypes: i) Epstein-Barr virus positive tumors, presenting recurrent PIK3CA mutations, extreme DNA hypermethylation and amplification of JAK2, PD-L1 and PD-L2; ii) microsatellite unstable tumors, characterized by elevated mutational rates; iii) genomically stable tumors, enriched for the diffuse-type and iv) tumors with chromosomal instability, the most common in the intestinal-type, showing marked aneuploidy and focal amplification of receptor tyrosine kinases [17].

Analyses using online database available differentially expressed genes have been identified between these two types. Intestinal-type samples overexpressed ATPIF1, PRDX2, PRKAR2A, and SMC1A, while diffuse-type samples overexpressed DTNA, GPR161, IDS, RHOQ, and TSHZ2. These two groups of genes demonstrated distinct prognoses [22].

There are plenty of data showing differences between intestinal and diffuse molecular features [18-20] including new experimental models, as organoid tumors, presenting typical molecular profiles according to the primary tumors from where they were developed [21]. An IARC publication deeply described molecular differences among intestinal and diffuse type gastric cancer, including the sequential molecular steps supposed to be needed for each cancer type to develop [23]. Additionally, both the Asian molecular classification [24], and TCGA classification [17] highlighted the relationship among the new molecular subtypes and Lauren's classification. Accordingly, intestinal and diffuse GAC present peculiar molecular profiles, suggesting the existence of different carcinogenic pathways distinguishing each cancer type.

\section{H. pylori infection and gastric acidity}

The relationship of gastric acidity and $H$. pylori infection is well known, since this bacterium can survive, and infect gastric mucosa, mainly in distal stomach, and cause peptic diseases, in a very acid environment. Nevertheless, regarding GAC, the supposed mechanism for $H$. pylori related carcinogenesis involves stomach corpus atrophy, instead of antrum infection, and inversely, results in reduction acidity.

According to the Correa hypothesis, gastric corpus atrophy following $H$. pylori infection, which results in increasing stomach $\mathrm{pH}$, may evolve to intestinal metaplasia, dysplasia and GAC, and might, partially, explain the intestinal type GAC [25]. Diffuse type carcinogenesis does not seem to be covered by this hypothesis, although there are reports relating $H$. pylori to both types of GAC.

Additionally to gastric atrophy, the huge utilization of medicines [26] that results in reduction of stomach acid secretion might contribute to generate a microenvironment known to be related to GAC carcinogenesis: gastric acidity reduction. The role of such medicines in contributing to an environment prone to GAC development remains under debate, since prolonged medical inhibition of acid secretion, per se, might not be enough to trigger GAC carcinogenesis (Fig. 1). Mainly, the eventual contribution of these medicines to diffuse type GAC remains insufficiently explored. Table 1 presents comparatively the characteristics of the histological types of the Lauren.

\section{The gastric microbiota}

Historically the stomach was thought to be a hostile sterile cavity, where microorganisms could not survive. H. pylori infection was first described in the 1980s. Recently, mainly due to new sequencing technologies, it was possible to uncover a wide variety of microorganisms in the human stomach [27].

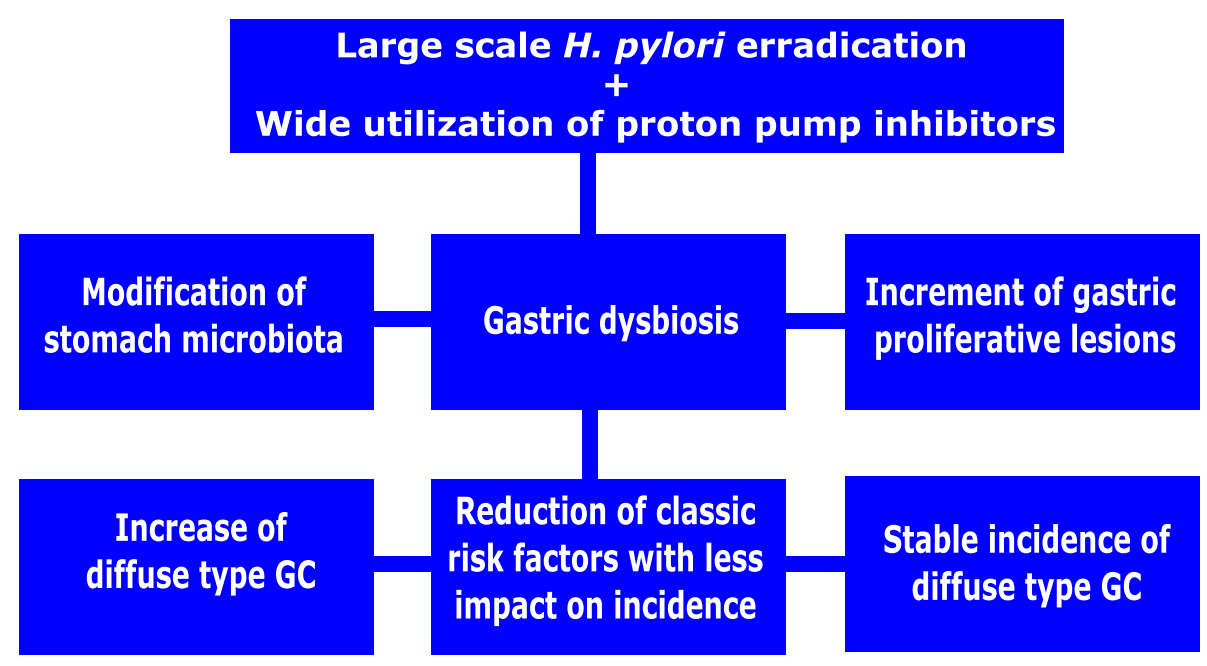

Fig. 1 Hypothesis to explain the continuing stable incidence of diffuse-type gastric cancer 
Table 1 Differences between Lauren's intestinal and diffuse types

\begin{tabular}{lll}
\hline & Lauren's Types Gastric Adenocarcinoma & Diffuse \\
\hline Characteristic & Intestinal & stable or increasing \\
Incidence trend & declining & lower \\
Prevalence in endemic areas & higher & weak \\
Environmental influence & strong & young \\
Age of occurrence & elderly & strong \\
Genetic factors & weak & $1: 1$ \\
Male/female ratio & $2: 1$ & proximal \\
Location of tumor & distal & tumour spreads along the gastric wall \\
Macroscopic growth pattern & growth into the lumen & unknown \\
Carcinogenesis & well known & mainly genomically stable (GS) \\
TCGA subtypes & mainly chromosomal instability (CIN) & worse \\
Prognosis & better & \\
\hline
\end{tabular}

As demonstrated for other tumor types, a gastric dysbiosis appears to take place during gastric carcinogenesis [27] and this diversity of microorganisms may differ between tumor subtypes, pointing to new causal agents. Gastric microbiota might potentially play a role in chemotherapy response, as shown to other tumors, and bacterial biotypes might serve as surrogate markers of a pre-neoplastic environment.

Whereas microbiome differences between intestinaland diffuse-types have not been largely explored, it is possible that differences exist, and could be considered for clinical intervention. Nevertheless, the importance of gastric microbiota on cancer incidence and even on benign diseases remains uncertain [27].

Treatment of $H$. pylori infection is based on combined antibiotic therapy, associated with proton-pump inhibitors; the use of this class of drugs appears to impact the gastric microbiota [28]. Several consequences of this treatment could be hypothesized: i) reduction of non- $H$. pylori bacteria sensitive to the antibiotics; ii) increased proliferation of bacteria resistant to treatment; iii) a replacement of the microbiota due to empty niches previously occupied by antibiotic-sensitive bacteria and, ultimately, iv) a dysbiotic stomach microbiota [27, 28].

Preliminary data seem to demonstrate that in the presence of $H$. pylori infection, the diversity of other bacteria is lower than in its absence [27]. The impact of these important alterations in GC, including the diffuse-type, is unknown. Although $H$. pylori infection appears to have a key role in gastric cancer, interactions with other bacteria may be part of the carcinogenic process [27], and the understanding of these interactions might shed light on the epidemiologic trends of the diffuse-type GAC.

Gastric H. pylori infection is recognized as the main cause of both gastric cancer [14] and duodenal peptic ulcer. Although the etiologic agent is the same, peptic ulcer occurs in a very acid environment, and the antrum seems to be the most important site of infection [25]. On the other hand, patients with duodenal peptic ulcer have a lower risk of GAC than that of the general population [14].

Interactions among different bacteria and $\mathrm{H}$. pylori infection may influence certain molecular pathways (Fig. 2). According to hypothetical scenarios: i) $H$. pylori infection plus specific bacterial co-infection may favor gastric cancer pathway; ii) H. pylori infection plus other specific bacteria may favor the benign peptic disease pathway; iii) treatment of $H$. pylori infection may favor the proliferation of specific bacteria that may have a role in diffuse-type carcinogenesis, thus counteracting the $H$. pylori eradication and keeping elevated the rates of diffuse-type cancers related to stomach infection.

\section{Genetic predisposition}

A genome-wide association study (GWAS) performed in a Japanese population identified a strong correlation between a particular single nucleotide polymorphism (SNP) rs2294008 (Met1Thr) in the Prostate Stem Cell Antigen gene (PSCA) and diffuse-type GC. The same group showed that a functional SNP in mucin 1 determines higher susceptibility to diffuse-type gastric cancer [29]. Nevertheless, among Caucasians, an association between variations in the promoter region of PSCA and GC risk has been also reported. However, for this population, the SNP rs2294008 variant carried a similar risk for diffuse and intestinal GAC types [30].

\section{Interfering on diffuse-type cancer burden}

Several potential strategies to control gastric cancer burden have been suggested, of which the following are the most commonly adopted: i) treatment of $H$. pylori infection [12, 13]; ii) avoidance of salt-preserved food [9]; and iii) surveillance of patients at risk (gastric cancer relatives, patients with intestinal metaplasia, and hereditary 


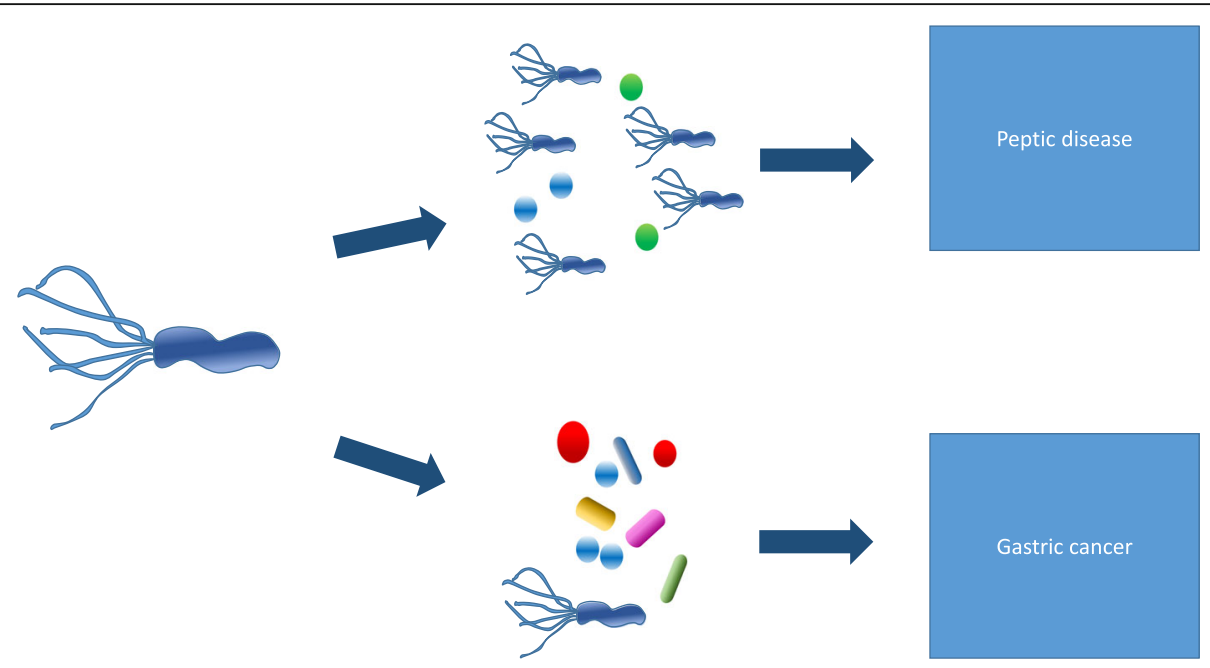

Fig. 2 The microbiota favors the evolution to either peptic disease or gastric cancer

cancer syndromes) [13]. However, except for the diffuse hereditary gastric cancer syndrome, which occurs in very few patients and is mainly associated with $C D H 1$ mutations, preventive strategies have not been effective in reducing the incidence of diffuse-type tumors.

Unlike metaplasia of the intestinal-type GAC, there are no pre-neoplastic lesions described for diffuse-type GAC that would allow surveillance or trigger preventive measures. As a consequence, innovative strategies are needed to tackle diffuse-type GAC prevention.

Looking outside the "cancer box", an interesting phenomenon in the stomach mucosa is the increasing incidence of hyperplastic lesions [31]. Notwithstanding its benign nature, hyperplastic lesions entail high risk DNA alterations that, associated with exposure to new carcinogenic agents and to microenvironment modifications of the stomach's mucosa, might favor a new pathway for diffuse GAC development.

Similarly to what happens regarding metaplasia and intestinal-type cancer risk, the vast majority of hyperplastic lesions will never develop as a diffuse-type cancer. Efforts to identify molecular aberrations present both in hyperplastic and other types of mucosa alterations should be investigated, in order to understand its role in diffuse-type gastric cancer.

Despite not being a consensus in current clinical practice, the identification of molecular biomarkers implicated in diffuse gastric cancer risk must be attempted. The main reason for optimism is the knowledge that molecular alterations occur many years (usually $>20$ years) before cancer occurrence and that significant technology improvements seen in recent years bode well for scientific breakthroughs in this field.

In this sense it is strongly recommended the implementation of investigations addressing the role of the gastric microbiota role in gastric cancer carcinogenesis the continuous monitoring of epidemiologic trends, including differences between intestinal and diffuse GAC types.

The possible connections among $H$. pylori infection, use of antibiotics, proton pump inhibitors, and other medications, including nonsteroidal anti-inflammatory drugs, that also modify the gastric microenvironment by interfering in gastric mucus barrier, should be carefully vetted. It also deserves attention the emergence of stomach lesions such as hyperplasic polyps, and also neuroendocrine gastric tumors, as well as the more recent diet modifications, as all these may fill central knowledge gaps, and contribute to understanding of carcinogenesis process involved in causing diffuse gastric cancer.

\section{Conclusion}

Diffuse-type GAC differs from the intestinal-type and has unexplained and underexplored characteristics. Among these are the decline in intestinal-type incidence not accompanied by a similar decline of diffuse cancers; the fact that the impact of reducing $H$. pylori gastric infection does not seem to be as effective for the diffuse as for intestinaltype; the widespread use of proton pump inhibitors and antibiotics, which may affect the diffuse-type carcinogenic pathways; the recent increase in benign proliferative stomach lesions that could favor diffuse-type carcinogenesis; and, of great importance, modifications in gastric microbiota and the microenvironment. The knowledge of the role of these events may allow the development of innovative strategies to control gastric cancer burden.

In order to reduce the incidence of diffuse gastric cancer, it will be necessary to increase the knowledge of the carcinogenesis of this histological type, enabling the discovery of etiological factors to be fought and the recognition of early stages susceptible to effective detection and treatment. 


\section{Abbreviations}

GAC: Gastric adenocarcinoma; GC: Gastric cancer; H. pylori: Helicobacter pylori; TCGA: The Cancer Genome Atlas; PIK3CA: Phosphatidylinositol-4,5biphosphate 3-kinase catalytic subunit alpha; DNA: Deoxyribonucleic acid; JAK2: Janus kinase 2; PD-L1: Programmed death-ligand 1; PD-

L2: Programmed death-ligand 2; ATPIF1: ATPase inhibitory factor 1; ATP: Adenosine triphosphate; PRDX2: Peroxiredoxin 2 gene; PRKAR2A: Protein kinase CAMP-dependent type II regulatory subunit alpha gene; SMC1A: Structural maintenance of chromosomes 1A gene; DTNA: Dystrobrevin alpha gene; GPR161: G protein-coupled receptor 161 gene; IDS: Iduronate 2-sulfatase gene; RHOQ: Ras homolog family member $\mathrm{Q}$ gene; TSHZ2: Teashirt zinc finger homeobox 2 gene; IARC: International Agency for Research on Cancer; GWAS: Genome-wide association study; SNP: Single nucleotide polymorphism; PSCA: Prostate stem cell antigen gene; CDH1: Cadherin-1 gene

\section{Acknowledgements}

We acknowledge Universidade Federal do Pará (PROPESP and FADESP) for technical support and Conselho Nacional de Desenvolvimento Científico e Tecnológico (CNPq) and Coordenação de Aperfeiçoamento de Pessoal de Nível Superior (CAPES) for fellowship support.

\section{Authors' contributions}

PPA, WFB and GI conceived the manuscript. PPA, WFB, GI, LGVC, FJFC, HCF, EDN, MCC and MS wrote the text. All authors have read and approved the manuscript in its final state.

\section{Funding}

not applicable

\section{Availability of data and materials}

not applicable.

\section{Ethics approval and consent to participate not applicable}

\section{Consent for publication}

not applicable.

\section{Competing interests}

none of the authors have any competing interests.

\section{Author details}

${ }^{1}$ Núcleo de Pesquisas em Oncologia, Universidade Federal do Pará, Belém, Pará, Brazil. ${ }^{2}$ Serviço de Cirurgia, Hospital Universitário João de Barros Barreto, Universidade Federal do Pará, Belém, Pará, Brazil. ${ }^{3}$ Universidade Federal de Minas Gerais, Belo Horizonte, Minas Gerais, Brazil. ${ }^{4}$ Department of Abdominal Surgery, A.C.Camargo Cancer Center, São Paulo, SP, Brazil. ${ }^{5}$ Departament of Clinical Oncology, A.C.Camargo Cancer Center, São Paulo, Brazil. ' Medical Genomics Laboratory, CIPE/A.C.Camargo Cancer Center, São Paulo, Brazil. ${ }^{7}$ Division of Cancer Epidemiology and Genetics, National Cancer Institute, National Institutes of Health, Rockville, MD, USA. ${ }^{8}$ Department of Epidemiology, Johns Hopkins Bloomberg School of Public Health, Baltimore, MD, USA.

Received: 2 July 2019 Accepted: 22 June 2020

Published online: 13 July 2020

\section{References}

1. Bray F, Ferlay J, Soerjomataran I, Siegel RL, Torre LA, Jemal A. Global cancer statistics 2018: GLOBOCAN estimates of incidence and mortality worldwide for 36 cancers in 185 countries. CA Cancer J Clin. 2018;68(6):394-424.

2. Rawla P, Barsouk A. Epidemiology of gastric cancer: global trends, risk factors and prevention. Prz Gastroenterol. 2019;14(1):26-8

3. Waldum HL, Fossmark R. Types of gastric carcinomas. Int J Mol Sci. 2018; 19(12):4109.

4. Wu H, Rusiecki JA, Zhu K, Potter J, Devesa SS. Stomach carcinoma incidence patterns in the United States by histologic type and anatomic site. Cancer Epidemiol Biomark Prev. 2009 Jul;18(7):1945-52.

5. Wachtel MS, Zhang Y, Chiriva-Internati M, Frezza EE. Different regression equations relate age to the incidence of Lauren types 1 and 2 stomach cancer in the SEER database: these equations are unaffected by sex or race. BMC Cancer. 2006:6:65.

6. Lee E, Liu L, Zhang J, et al. Stomach Cancer disparity among Korean Americans by tumor characteristics: comparison with non-Hispanic whites, Japanese Americans, south Koreans, and Japanese. Cancer Epidemiol Biomark Prev. 2017 Apr;26(4):587-96.

7. Plummer M, Franceschi S, Vignat J, Forman D, de Martel C. Global burden of gastric cancer attributable to helicobacter pylori. Int J Cancer. 2015;136(2): 487-90

8. Petrelli F, Berenato $R$, Turati $L$, et al. Prognostic value of diffuse versus intestinal histotype in patients with gastric cancer: a systematic review and meta-analysis. J Gastrointest Oncol. 2017;8(1):148-63.

9. Bonequi P, Meneses-González F, Correa P, et al. Risk factors for gastric cancer in Latin America: a meta-analysis. Cancer Causes Control. 2013;24(2): 217-31.

10. Wang $Q$, Chen $Y$, Wang $X$, et al. Consumption of fruit, but not vegetables, may reduce risk of gastric cancer: results from a meta-analysis of cohort studies. Eur J Cancer. 2014;50(8):1498-509.

11. Crowe SE. Helicobacter pylori infection. N Engl J Med. 2019:380(12):1158-65.

12. Choi IJ, Kook MC, Kim Yl, et al. Helicobacter pylori therapy for the prevention of Metachronous gastric Cancer. N Engl J Med. 2018:378(12): 1085-95.

13. Choi IJ, Kim CG, Lee JY, Kim YI, Kook MC, Park B, Joo J. Family history of gastric Cancer and helicobacter pylori treatment. N Engl J Med. 2020;382(5): 427-36.

14. de Assumpção PP, Araújo TMT, de Assumpção PB, Barra WF, et al. Suicide journey of $\mathrm{H}$. pylori through gastric carcinogenesis: the role of non-H. pylori microbiome and potential consequences for clinical practice. Eur J Clin Microbiol Infect Dis. 2019;38(9):1591-7.

15. Take S, Mizuno M, Ishiki K, et al. Risk of gastric cancer in the second decade of follow-up after helicobacter pylori eradication. J Gastroenterol. 2020;55(3): 281-8.

16. Inoue M. Changing epidemiology of helicobacter pylori in Japan. Gastric Cancer. 2017:20:3-7.

17. Cancer Genome Atlas Research Network. Comprehensive molecular characterization of gastric adenocarcinoma. Nature. 2014;513(7517):202-9.

18. Kim SK, Kim HJ, Park JL, Heo H, Kim SY, Lee SI, Song KS, Kim WH, Kim YS. Identification of a molecular signature of prognostic subtypes in diffusetype gastric cancer. Gastric Cancer. 2020;23(3):473-82.

19. Oue N, Sentani K, Sakamoto N, Uraoka N, Yasui W. Molecular carcinogenesis of gastric cancer: Lauren classification, mucin phenotype expression, and câncer stem cells. Int J Clin Oncol. 2019;24(7):771-8.

20. Binato R, Santos EC, Boroni M, Demachki S, Assumpção P, Abdelhay E. A common molecular signature of intestinal-type gastric carcinoma indicates processes related to gastric carcinogenesis. Oncotarget. 2017; 9(7):7359-71.

21. Yan HHN, Siu HC, Law S, et al. A Comprehensive Human Gastric Cancer Organoid Biobank Captures Tumor Subtype Heterogeneity and Enables Therapeutic Screening. Cell Stem Cell. 2018;23(6):882-97 e11.

22. Zhang C, Min L, Liu J, et al. Integrated analysis identified an intestinal-like and a diffuse-like gene sets that predict gastric cancer outcome. Tumor Biol. 2016;37(12):16317-35.

23. Tahara E. Genetic pathways of two types of gastric cancer. IARC Sci Publ. 2004;157:327-49.

24. Cristescu R, Lee J, Nebozhyn M, et al. Molecular analysis of gastric cancer identifies subtypes associated with distinct clinical outcomes. Nat Med. 2015:21(5):449-56

25. Correa P, Houghton J. Carcinogenesis of helicobacter pylori. Gastroenterology. 2007:133(2):659-72.

26. Haastrup P, Paulsen MS, Zwisler JE, et al. Rapidly increasing prescribing of proton pump inhibitors in primary care despite interventions: a nationwide observational study. Eur J Gen Pract. 2014;20(4):290-3.

27. Wang LL, Yu XJ, Zhan SH, Jia SJ, Tian ZB, Dong QJ. Participation of microbiota in the development of gastric cancer. World J Gastroenterol. 2014;20(17):4948-52.

28. Imhann F, Vich Vila A, Bonder MJ, et al. The influence of proton pump inhibitors and other commonly used medication on the gut microbiota. Gut Microbes. 2017:8(4):351-8.

29. Saeki N, Saito A, Choi IJ, et al. A functional single nucleotide polymorphism in mucin 1, at chromosome 1q22, determines susceptibility to diffuse-type gastric cancer. Gastroenterology. 2011;140(3):892-902. 
30. Sala N, Muñoz X, Travier N, et al. Prostate stem-cell antigen gene is associated with diffuse and intestinal gastric cancer in Caucasians: results from the EPIC-EURGAST study. Int J Cancer. 2012;130(10):2417-27.

31. Cao H, Wang B, Zhang Z, et al. Distribution trends of gastric polyps: an endoscopy database analysis of 24121 northern Chinese patients. J Gastroenterol Hepatol. 2012;27(7):1175-80.

\section{Publisher's Note}

Springer Nature remains neutral with regard to jurisdictional claims in published maps and institutional affiliations.

Ready to submit your research? Choose BMC and benefit from:

- fast, convenient online submission

- thorough peer review by experienced researchers in your field

- rapid publication on acceptance

- support for research data, including large and complex data types

- gold Open Access which fosters wider collaboration and increased citations

- maximum visibility for your research: over $100 \mathrm{M}$ website views per year

At BMC, research is always in progress.

Learn more biomedcentral.com/submissions 